\title{
On Kakeya Conditions for Achievement Sets
}

\author{
Jacek Marchwicki@ and Piotr Miska(D)
}

\begin{abstract}
We prove that for each infinite subset $C$ of $\mathbb{N}$ there exists a sequence $\left(x_{n}\right)$ such that $\left\{n: x_{n}>r_{n}\right\}=C$ and the achievement set $A\left(x_{n}\right)$ is a Cantor set. Moreover, we show that it is possible to construct a sequence $\left(x_{n}\right)$ such that the set $\left\{n: x_{n}>r_{n}\right\}$ has asymptotic density $\alpha$ for each $\alpha \in[0,1)$ and $A\left(x_{n}\right)$ is a Cantorval.
\end{abstract}

Mathematics Subject Classification. Primary: 40A05, Secondary: 11K31.

Keywords. Achievement set, set of subsums, absolutely convergent series, Cantor set, Cantorval, asymptotic density, purely atomic measure.

\section{Preliminaries}

Let $\mathbb{N}=\{1,2,3,4, \ldots\}$ and $\mathbb{N}_{0}=\{0,1,2,3,4, \ldots\}$ denote the sets of positive and non-negative integers, respectively.

An achievement set is a set of the form $A\left(x_{n}\right)=\left\{\sum_{n=1}^{\infty} \varepsilon_{n} x_{n}:\left(\varepsilon_{n}\right) \in\{0\right.$, $\left.1\}^{\mathbb{N}}\right\}=\left\{\sum_{n \in A} x_{n}: A \subset \mathbb{N}\right\}$, where $\sum_{n=1}^{\infty} x_{n}$ is convergent series of positive reals. We additionally assume that $x_{n} \geq x_{n+1}$ for every $n \in \mathbb{N}$.

The first paper where achievement set was considered is that of Kakeya, see [12]. The author presented sufficient conditions for achievement set to be interval or Cantor set in terms of series terms $x_{n}$ and the remainders $r_{n}=$ $\sum_{k>n} x_{k}$.

\section{Theorem 1.1 (Kakeya).}

(1) $A\left(x_{n}\right)$ is a closed interval if and only if $x_{n} \leq r_{n}$ for all $n$;

(2) $A\left(x_{n}\right)$ is homeomorphic to a Cantor set, if $x_{n}>r_{n}$ for all $n \in \mathbb{N}$.

The sequences $\left(x_{n}\right)$ which satisfy condition (1) are called interval-filling and were deeply studied in [6-8]. The interval-filling sequences $\left(x_{n}\right)$ are also 
known as slowly convergent, while the sequences satisfying condition (2) are called quickly or fast convergent.

Softened condition (1) ' $x_{n} \leq r_{n}$ for all but finitely many $n \in \mathbb{N}$ ' is equivalent to that $A\left(x_{n}\right)$ is a finite union of closed intervals. On the other hand, if $x_{n}>r_{n}$ for every $n>N$, then $A\left(x_{n}\right)=\Sigma+A\left(\left(x_{n}\right)_{n>N}\right)$ where $\Sigma=\left\{\sum_{n=1}^{N} \varepsilon_{n} x_{n}:\left(\varepsilon_{n}\right) \in\{0,1\}^{N}\right\}$. Since $\Sigma$ is finite and $A\left(\left(x_{n}\right)_{n>N}\right)$ is a Cantor set, then $A\left(x_{n}\right)$ as a finite union of Cantor sets is a Cantor set as well.

Kakeya claimed that for an absolutely convergent series with infinitely many nonzero terms the set $A\left(x_{n}\right)$ is either a finite union of closed intervals or a set homeomorphic to a Cantor set. Due to the Guthrie-Nymann characterization the achievement set is either a Cantor set or a finite union of intervals or a Cantorval.

Theorem 1.2. For an absolutely convergent series $\sum_{n=1}^{\infty} x_{n}$ with infinitely many nonzero terms, the set $A\left(x_{n}\right)$ is one of the following: a finite sum of closed intervals, homeomorphic to a Cantor set or a Cantorval, that is a set homeomorphic to $A\left(y_{n}\right)$ for $y_{2 n-1}=\frac{3}{4^{n}}$ and $y_{2 n}=\frac{2}{4^{n}}$ for all $n \in \mathbb{N}$.

It is known that a Cantorval is a nonempty compact set in $\mathbb{R}$ such that it is the closure of its interior and both endpoints of any nontrivial connected component are accumulation points of its trivial connected components. Another topological characterizations of Cantorvals can be found in [4,13]. Theorem 1.2 was first stated in [11] but its correct proof was given in [14]. The set $A\left(y_{n}\right)$ for $y_{2 n-1}=\frac{3}{4^{n}}$ and $y_{2 n}=\frac{2}{4^{n}}$ for all $n \in \mathbb{N}$ is called the GuthrieNymann Cantorval. A multigeometric sequence is a sequence of the form $\left(x_{n}\right)=\left(a_{1}, a_{2}, \ldots, a_{m} ; q\right)=\left(a_{1} q, a_{2} q, \ldots, a_{m} q, a_{1} q^{2}, a_{2} q^{2}, \ldots, a_{m} q^{2}, a_{1} q^{3}, \ldots\right)$, where $a_{1} \geq a_{2} \geq \cdots \geq a_{m}$ and $a_{1} q \leq a_{m}$ which ensure that $\left(x_{n}\right)$ is nonincreasing. Using that notion, the Guthrie and Nymann's Cantorval can be viewed as $A\left(3,2 ; \frac{1}{4}\right)$. The set $\Sigma=A\left(a_{1}, \ldots, a_{m}\right)=\left\{\sum_{n=1}^{m} \varepsilon_{n} a_{n}:\left(\varepsilon_{n}\right) \in\{0\right.$, $\left.1\}^{m}\right\}$ will be of special interest to us. Then $A\left(a_{1}, a_{2}, \ldots, a_{m} ; q\right)=\left\{\sum_{n=1}^{\infty} y_{n} q^{n}\right.$ $\left.:\left(y_{n}\right) \in \Sigma^{\mathbb{N}}\right\}$. Multigeometric series were studied in $[1,3,5]$.

To obtain Cantorval, both conditions $x_{n} \leq r_{n}$ and $x_{n}>r_{n}$ must be fulfilled, each for infinitely many $n$ 's. However, there are known series which fulfill both conditions but their achievement sets are Cantor sets. We ask if there are subsets $E$ of $\mathbb{N}$ such that the condition $x_{n} \leq r_{n} \Leftrightarrow n \in E$ implies that $A\left(x_{n}\right)$ is a Cantorval. Similarly, are there infinite subsets $E$ of $\mathbb{N}$ such that the condition $x_{n} \leq r_{n} \Leftrightarrow n \in E$ implies that $A\left(x_{n}\right)$ is a Cantor set? In the paper we present the negative answer for the first question (Theorem 2.1) and partially negative for the second one in the third section.

We will base on the following theorem proved in [5], which gives a simple sufficient condition for the achievement set to be a Cantorval.

Theorem 1.3. Let $a_{1} \geq a_{2} \geq \cdots \geq a_{m}$ be positive integers. Assume that there exist positive integers $n_{0}$ and $r$ such that $\Sigma \supset\left\{n_{0}, n_{0}+1, \ldots, n_{0}+r\right\}$. If $q \geq \frac{1}{r+1}$, then $A\left(a_{1}, a_{2}, \ldots, a_{m} ; q\right)$ has a nonempty interior. If $q<\frac{a_{m}}{\sum_{i=1}^{m} a_{i}+a_{m}}$, 
then $A\left(a_{1}, a_{2}, \ldots, a_{m} ; q\right)$ is not a finite union of intervals. Consequently, if $\frac{1}{r+1} \leq q<\frac{a_{m}}{\sum_{i=1}^{m} a_{i}+a_{m}}$, then $A\left(a_{1}, a_{2}, \ldots, a_{m} ; q\right)$ is a Cantorval.

It is worth to mention that the negative answer for Kakeya's conjecture was obtained before Guthrie and Nymann's paper. The first counterexample was given without proof by Weinstein and Shapiro in [16]. In [9] Ferens constructed a purely atomic finite measure $\mu$ and proved that its range is a Cantorval. The theories of achievement sets and purely atomic finite measures coincide. Indeed, we may assume that $\mu$ is defined on $\mathbb{N}$. Then $\operatorname{rng}(\mu)=\{\mu(A): A \subset \mathbb{N}\}=A\left(x_{n}\right)$, where the terms of our series are the values of measure on singletons, that is $x_{n}=\mu(\{n\})$ for all $n \in \mathbb{N}$. Hence, we may say that Ferens observed that $A\left(7,6,5,4,3 ; \frac{2}{27}\right)$ is a Cantorval. In the paper we base on two simple properties of the achievement sets. First of them is connected with the equality $A\left(\left|x_{n}\right|\right)=A\left(x_{n}\right)+\sum_{n=1}^{\infty} x_{n}^{-}$(recall that $x^{-}=-\min \{x, 0\}$ for each real number $\left.x\right)$, which allows us to consider only the sequences with all terms positive. The second property is that for any absolutely convergent series $\sum_{n=1}^{\infty} x_{n}$ and any bijection $\sigma: \mathbb{N} \rightarrow \mathbb{N}$ the equality $A\left(x_{\sigma(n)}\right)=A\left(x_{n}\right)$ holds. This means that the achievement set is invariant to any rearrangement of its terms and hence we may assume that the sequence $\left(x_{n}\right)$ is non-increasing. It is also needed to introduce the Kakeya conditions.

The conditions in which we consider the inequalities between the terms $x_{n}$ and the tails $r_{n}$ are also known as Kakeya or Kakeya-like conditions. In $[6,7]$ the authors considered a subset $U\left(x_{n}\right) \subset A\left(x_{n}\right)$ of those points which are obtained in a unique way. In other words, $x \in U\left(x_{n}\right)$ if and only if there exists a unique set $A$ or $0-1$ sequence $\left(\varepsilon_{n}\right)$ such that $x=\sum_{n \in A}^{\infty} x_{n}$ or $x=\sum_{n=1}^{\infty} \varepsilon_{n} x_{n}$, respectively. Clearly, $U\left(x_{n}\right) \supset\left\{0, \sum_{n=1}^{\infty} x_{n}\right\}$. The authors in [6,7] proved that if an interval-filling sequence is a locker, that is $x_{k} \leq r_{k+1}$ for all $k$, then $U\left(x_{n}\right)$ contains only the endpoints 0 and $\sum_{n=1}^{\infty} x_{n}$.

On the other hand, if the pure Kakeya condition Theorem 1.1(2) holds, then $U\left(x_{n}\right)=A\left(x_{n}\right)$, which can be found in [15]. Let us consider the case of $U\left(x_{n}\right)=A\left(x_{n}\right)$. Then, the mapping

$$
T:\{0,1\}^{\mathbb{N}} \ni\left(\varepsilon_{n}\right) \mapsto \sum_{n=1}^{\infty} \varepsilon_{n} x_{n} \in A\left(x_{n}\right) .
$$

is bijective. $T$ is also continuous with respect to the product topology on $\{0,1\}^{\mathbb{N}}$ and natural topology on $\mathbb{R}$ as the series $\sum_{n=1}^{\infty} x_{n}$ is absolutely convergent. The set $\{0,1\}^{\mathbb{N}}$ equipped with the product topology is homeomorphic to a Cantor set . In particular, $\{0,1\}^{\mathbb{N}}$ is a compact topological space. This means that $T$ is a homeomorphism. Thus, if $A\left(x_{n}\right)=U\left(x_{n}\right)$, then $A\left(x_{n}\right)$ is homeomorphic to a Cantor set.

Although the condition Theorem 1.1(1) characterizes the case when $A\left(x_{n}\right)$ is a finite union of closed intervals by Kakeya conditions, the implication in Theorem 1.1(2) can not be reversed. The authors showed in [10] that even the implication $\left(x_{n}\right)$ is fast convergent $\Rightarrow U\left(x_{n}\right)=A\left(x_{n}\right)$ is far from reversal and 
constructed the sequence $\left(x_{n}\right)$ for which $x_{2 n-1}<r_{2 n-1}$ for each $n \in \mathbb{N}$ and $U\left(x_{n}\right)=A\left(x_{n}\right)$. In the paper we improve their construction and show that the fast convergence condition may be broken in any non-cofinite set of indices, see Theorem 2.1. Note that by Theorem 1.1 (1) there are no such constructions for any cofinite set. There are several more results connected with the Kakeya conditions, in particular the three gaps lemmas, which can be found in [4].

From the Kakeya condition we see that if the series $\sum_{n=1}^{\infty} x_{n}$ is ,,closer" to satisfy the fast convergence condition, then its achievement set $A\left(x_{n}\right)$ is more likely to be a Cantor set. It is natural to ask if the form of achievement set $A\left(x_{n}\right)$ is somehow connected with the largeness of the set $\left\{n \in \mathbb{N}: x_{n}>r_{n}\right\}$. Probably the most common and popular function which describes the size of subsets of $\mathbb{N}$ is asymptotic density. Let us recall the notions of lower and upper asymptotic densities of subsets of $\mathbb{N}$ :

$$
\begin{array}{ll}
\underline{\delta}(F)=\liminf _{n \rightarrow \infty} \frac{|F \cap\{1,2, \ldots, n\}|}{n} & \text { (lower asymptotic density), } \\
\bar{\delta}(F)=\limsup _{n \rightarrow \infty} \frac{|F \cap\{1,2, \ldots, n\}|}{n} \quad \text { (upper asymptotic density). }
\end{array}
$$

If $\underline{\delta}(F)=\bar{\delta}(F)$, then we define asymptotic density of $F$ as the common value of lower and upper asymptotic density of $F$. The main purpose of the paper is to show that it is not possible to characterize or even give necessary either sufficient Kakeya-like condition for the form of $A\left(x_{n}\right)$ by using the asymptotic density of the set $\left\{n \in \mathbb{N}: x_{n}>r_{n}\right\}$. To avoid conflict of notations, in the whole paper we denote the terms of the series as $\left(x_{n}\right),\left(y_{n}\right)$ or $\left(z_{n}\right)$. The sequences of tails for the series $\sum_{n=1}^{\infty} x_{n}, \sum_{n=1}^{\infty} y_{n}$ and $\sum_{n=1}^{\infty} x_{n}$ are donted by $\left(r_{n}(\mathbf{x})\right),\left(r_{n}(\mathbf{y})\right)$ and $\left(r_{n}(\mathbf{z})\right)$, respectively. However, if it is known what series we consider, its sequence of tails will be denoted by $\left(r_{n}\right)$ for simplification of notation.

\section{Extreme Examples of Cantor Set as an Achievement Set}

We will show that for each (arbitrarily large) not cofinite subset $B$ of $\mathbb{N}$ one can construct a convergent series $\sum_{n=1}^{\infty} x_{n}$ with positive terms such that the fast convergence condition with respect to this series fails exactly on $B$ but the set $A\left(x_{n}\right)$ is a Cantor set.

Theorem 2.1. Let $C=\left\{n_{1}<n_{2}<n_{3}<\cdots\right\}$ be an infinite subset of $\mathbb{N}$. Then there exists a non-increasing sequence $\left(x_{n}\right)$ of positive real numbers such that the series $\sum_{n=1}^{\infty} x_{n}$ is convergent, $\left\{n \in \mathbb{N}: x_{n}>r_{n}\right\}=C,\left\{n \in \mathbb{N}: x_{n}<\right.$ $\left.r_{n}\right\}=C^{c}$ and $A\left(x_{n}\right)=U\left(x_{n}\right)$. In particular, $A\left(x_{n}\right)$ is a Cantor set.

Proof. Put $n_{-1}=n_{0}=0$ and for each $k \in \mathbb{N}_{0}$ define $y_{k}=3^{-k} \cdot 2^{-n_{k-1}} \prod_{j=0}^{k}$ $\left(n_{j+1}-n_{j}\right)^{-1}$. Then, for $k \in \mathbb{N}_{0}$ and $i \in\left\{1, \ldots, n_{k+1}-n_{k}\right\}$ we define $x_{n_{k}+i}=$ $y_{k}+2^{-i} y_{k+1}$. In other words, if $n_{k}<n \leq n_{k+1}$, then $x_{n}=y_{k}+2^{n_{k}-n} y_{k+1}$. 
It is clear, that for each $k \in \mathbb{N}_{0}$ and $i \in\left\{1, \ldots, n_{k+1}-n_{k}-1\right\}$ we have $x_{n_{k}+i}>x_{n_{k}+i+1}$. Let us check that for any $k \in \mathbb{N}$ we have $x_{n_{k}}>x_{n_{k}+1}$ :

$$
\begin{aligned}
x_{n_{k}}= & x_{n_{k-1}+n_{k}-n_{k-1}}=y_{k-1}+2^{n_{k-1}-n_{k}} y_{k}=3^{-k+1} \cdot 2^{-n_{k-2}} \prod_{j=0}^{k-1}\left(n_{j+1}-n_{j}\right)^{-1} \\
& +3^{-k} \cdot 2^{-n_{k}} \prod_{j=0}^{k}\left(n_{j+1}-n_{j}\right)^{-1}>3^{-k} \cdot 2^{-n_{k-1}} \prod_{j=0}^{k}\left(n_{j+1}-n_{j}\right)^{-1} \\
& +3^{-k-1} \cdot 2^{-n_{k}-1} \prod_{j=0}^{k+1}\left(n_{j+1}-n_{j}\right)^{-1}=y_{k}+2^{-1} y_{k+1}=x_{n_{k}+1} .
\end{aligned}
$$

We show that the series $\sum_{n=1}^{\infty} x_{n}$ is convergent:

$$
\begin{aligned}
\sum_{n=1}^{\infty} x_{n} & =\sum_{k=0}^{\infty} \sum_{i=1}^{n_{k+1}-n_{k}} x_{n_{k}+i}=\sum_{k=0}^{\infty} \sum_{i=1}^{n_{k+1}-n_{k}}\left(y_{k}+2^{-i} y_{k+1}\right) \\
& <\sum_{k=0}^{\infty}\left[\left(n_{k+1}-n_{k}\right) y_{k}+y_{k+1}\right] \\
& <\sum_{k=0}^{\infty}\left(3^{-k}+3^{-k-1}\right)=\frac{1+\frac{1}{3}}{1-\frac{1}{3}}=2 .
\end{aligned}
$$

If $k \in \mathbb{N}_{0}$ and $i \in\left\{1, \ldots, n_{k+1}-n_{k}-1\right\}$, then

$$
\begin{aligned}
x_{n_{k}+i} & =y_{k}+2^{-i} y_{k+1}<y_{k}+2^{-i-1} y_{k+1}+y_{k+1}+2^{-1} y_{k+2} \\
& =x_{n_{k}+i+1}+x_{n_{k+1}+1}<r_{n_{k}+i} .
\end{aligned}
$$

Let us notice that for $k \in \mathbb{N}_{0}$ we have

$$
2^{n_{k-1}-n_{k}} y_{k}>r_{n_{k+1}}+y_{k+1} \text {. }
$$

Indeed,

$$
\begin{aligned}
r_{n_{k+1}} & +y_{k+1} \\
& =\sum_{l=k+1}^{\infty} \sum_{i=1}^{n_{l+1}-n_{l}}\left(y_{l}+2^{-i} y_{l+1}\right)+y_{k+1}<\sum_{l=k+1}^{\infty}\left[\left(n_{l+1}-n_{l}\right) y_{l}+y_{l+1}\right]+y_{k+1} \\
& =\sum_{l=k+1}^{\infty}\left(n_{l+1}-n_{l}+1\right) y_{l} \\
& =\sum_{l=k+1}^{\infty}\left[\left(n_{l+1}-n_{l}+1\right) \cdot 3^{-l} \cdot 2^{-n_{l-1}} \prod_{j=0}^{l}\left(n_{j+1}-n_{j}\right)^{-1}\right] \\
& =3^{-k} \cdot 2^{-n_{k}} \sum_{l=k+1}^{\infty}\left[\left(1+\frac{1}{n_{l+1}-n_{l}}\right) \cdot 3^{k-l} \cdot 2^{n_{k}-n_{l-1}} \prod_{j=0}^{l-1}\left(n_{j+1}-n_{j}\right)^{-1}\right]
\end{aligned}
$$




$$
\begin{aligned}
& <3^{-k} \cdot 2^{-n_{k}} \sum_{l=k+1}^{\infty}\left[2 \cdot 3^{k-l} \prod_{j=0}^{k}\left(n_{j+1}-n_{j}\right)^{-1}\right] \\
& =2^{n_{k-1}-n_{k}} \cdot 3^{-k} \cdot 2^{-n_{k-1}} \prod_{j=0}^{k}\left(n_{j+1}-n_{j}\right)^{-1} \sum_{l=k+1}^{\infty} 2 \cdot 3^{k-l} \\
& =2^{n_{k-1}-n_{k}} y_{k} \cdot \frac{\frac{2}{3}}{1-\frac{1}{3}}=2^{n_{k-1}-n_{k}} y_{k} .
\end{aligned}
$$

In particular,

$$
\begin{aligned}
x_{n_{k}}= & x_{n_{k-1}+\left(n_{k}-n_{k-1}\right)}=y_{k-1}+2^{n_{k-1}-n_{k}} y_{k}>y_{k-1}+y_{k+1}+r_{n_{k+1}} \\
= & 3 \cdot 2^{n_{k-1}-n_{k-2}}\left(n_{k+1}-n_{k}\right) y_{k}+y_{k+1}+r_{n_{k+1}}>\sum_{i=1}^{n_{k+1}-n_{k}}\left(y_{k}+2^{-i} y_{k+1}\right) \\
& +r_{n_{k+1}}=\sum_{i=1}^{n_{k+1}-n_{k}} x_{n_{k}+i}+r_{n_{k+1}}=r_{n_{k}}
\end{aligned}
$$

for each $k \in \mathbb{N}$, where the first inequality follows from (1). As a result, if $n \in C$, then $x_{n}>r_{n}$, and if $n \in C^{c}$, then $x_{n}<r_{n}$.

To the end of the proof it is enough to show that if $F, G \subset \mathbb{N}$ and $\sum_{n \in F} x_{n}=\sum_{n \in G} x_{n}$, then $F=G$. Denote $I_{k}=\left\{n_{k}+1, \ldots, n_{k+1}\right\}$ for each $k \in \mathbb{N}_{0}$. We will show by induction on $k$ that $F \cap I_{k}=G \cap I_{k}$. Assume that $F \cap I_{l}=G \cap I_{l}$ for all $l<k$. First, let us note that $\left|F \cap I_{k}\right|=\left|G \cap I_{k}\right|$. Otherwise, we would have

$$
\begin{aligned}
& \left|\sum_{n \in F} x_{n}-\sum_{n \in G} x_{n}\right|=\mid \sum_{n \in F \cap I_{k}} y_{k}-\sum_{n \in G \cap I_{k}} y_{k} \\
& \quad+\sum_{n \in F \cap I_{k}} 2^{n_{k}-n} y_{k+1}-\sum_{n \in G \cap I_{k}} 2^{n_{k}-n} y_{k+1} \\
& \quad+\sum_{\substack{n \in F \\
n>n_{k+1}}} x_{n}-\sum_{\substack{n \in G \\
n>n_{k+1}}} x_{n}|\geq| \sum_{n \in F \cap I_{k}} y_{k}-\sum_{n \in G \cap I_{k}} y_{k} \mid \\
& \quad-\left|\sum_{n \in F \cap I_{k}} 2^{n_{k}-n} y_{k+1}-\sum_{n \in G \cap I_{k}} 2^{n_{k}-n} y_{k+1}\right|-\left|\sum_{\substack{n \in F \\
n>n_{k+1}}} x_{n}-\sum_{\substack{n \in G \\
n>n_{k+1}}} x_{n}\right| \\
& >y_{k}-y_{k+1}-r_{n_{k+1}}>0,
\end{aligned}
$$


where the last inequality follows form (1). Next, suppose that $\left|F \cap I_{k}\right|=\left|G \cap I_{k}\right|$ but $F \cap I_{k} \neq G \cap I_{k}$. Then $\left|\sum_{n \in F \cap I_{k}} x_{n}-\sum_{n \in G \cap I_{k}} x_{n}\right| \in\left[2^{n_{k}-n_{k+1}} y_{k+1} ; 2^{-1}\right.$ $\left.y_{k+1}\right]$. If $\left|F \cap I_{k+1}\right| \neq\left|G \cap I_{k+1}\right|$, then

$$
\begin{aligned}
& \left|\sum_{n \in F} x_{n}-\sum_{n \in G} x_{n}\right|=\mid \sum_{n \in F \cap I_{k}} x_{n}-\sum_{n \in G \cap I_{k}} x_{n}+\sum_{n \in F \cap I_{k+1}} y_{k+1}-\sum_{n \in G \cap I_{k+1}} y_{k+1} \\
& +\sum_{n \in F \cap I_{k+1}} 2^{n_{k+1}-n} y_{k+2}-\sum_{n \in G \cap I_{k+1}} 2^{n_{k+1}-n} y_{k+2}+\sum_{\substack{n \in F \\
n>n_{k+2}}} x_{n}-\sum_{\substack{n \in G \\
n>n_{k+2}}} x_{n} \\
& \geq\left|\sum_{n \in F \cap I_{k+1}} y_{k+1}-\sum_{n \in G \cap I_{k+1}} y_{k+1}\right|-\left|\sum_{n \in F \cap I_{k}} x_{n}-\sum_{n \in G \cap I_{k}} x_{n}\right| \\
& -\left|\sum_{n \in F \cap I_{k+1}} 2^{n_{k+1}-n} y_{k+2}-\sum_{n \in G \cap I_{k+1}} 2^{n_{k+1}-n} y_{k+2}\right| \\
& -\left|\sum_{\substack{n \in F \\
n>n_{k+2}}} x_{n}-\sum_{\substack{n \in G \\
n>n_{k+2}}} x_{n}\right|>y_{k+1}-2^{-1} y_{k+1}-y_{k+2}-r_{n_{k+2}} \\
& =2^{-1} y_{k+1}-y_{k+2}-r_{n_{k+2}}>0 \text {, }
\end{aligned}
$$

where the last inequality follows form (1) applied for $k+1$ inserted in the place of $k$. If $\left|F \cap I_{k+1}\right|=\left|G \cap I_{k+1}\right|$, then

$$
\begin{aligned}
& \left|\sum_{n \in F} x_{n}-\sum_{n \in G} x_{n}\right|=\mid \sum_{n \in F \cap I_{k}} x_{n}-\sum_{n \in G \cap I_{k}} x_{n}+\sum_{n \in F \cap I_{k+1}} y_{k+1}-\sum_{n \in G \cap I_{k+1}} y_{k+1} \\
& \quad+\sum_{n \in F \cap I_{k+1}} 2^{n_{k+1}-n} y_{k+2}-\sum_{n \in G \cap I_{k+1}} 2^{n_{k+1}-n} y_{k+2}+\sum_{\substack{n \in F \\
n>n_{k+2}}} x_{n}-\sum_{\substack{n \in G \\
n>n_{k+2}}} x_{n} \mid \\
& \geq\left|\sum_{n \in F \cap I_{k}} x_{n}-\sum_{n \in G \cap I_{k}} x_{n}\right|-\left|\sum_{n \in F \cap I_{k+1}} 2^{n_{k+1}-n} y_{k+2}-\sum_{n \in G \cap I_{k+1}} 2^{n_{k+1}-n} y_{k+2}\right| \\
& \quad-\left|\sum_{\substack{n \in F \\
n>n_{k+2}}} x_{n}-\sum_{\substack{n \in G \\
n>n_{k+2}}} x_{n}\right| \\
& >2^{n_{k}-n_{k+1}} y_{k+1}-y_{k+2}-r_{n_{k+2}}>0,
\end{aligned}
$$

where, once again, the last inequality follows form (1) applied for $k+1$ inserted in the place of $k$. Finally, there must be $F \cap I_{k}=G \cap I_{k}$ for each $k \in \mathbb{N}_{0}$. As a result, $F=\bigcup_{k=0}^{\infty}\left(F \cap I_{k}\right)=\bigcup_{k=0}^{\infty}\left(G \cap I_{k}\right)=G$. This means that every element of the set $A\left(x_{n}\right)$ can be uniquely represented as a subsum of the series $\sum_{n=1}^{\infty} x_{n}$. 
Corollary 2.2. For each $0 \leq \alpha \leq \beta \leq 1$ there exists a convergent series $\sum_{n=1}^{\infty} x_{n}$ with positive terms such that $A\left(x_{n}\right)=U\left(x_{n}\right)$ and the set $\{n \in \mathbb{N}$ : $\left.x_{n}>r_{n}\right\}$ has lower asymptotic density equal to $\alpha$ and upper one equal to $\beta$.

\section{Family of Cantorvals}

To simplify the notation when we will write $[a, b]$ instead of $\{a, a+1, a+$ $2, \ldots, b\}$.

Lemma 3.1. The following equality holds for all $n, k \in \mathbb{N}$.

$$
\Sigma_{n, k}:=A\left(\left\{2^{p}: p \in[1, n+k]\right\} \cup\left\{2^{n}+1\right\}\right)=B_{n, k} \cup M_{n, k} \cup F_{n, k},
$$

where $B_{n, k}:=\left\{2 p: p \in\left[0,2^{n-1}-1\right]\right\}, M_{n, k}:=\left[2^{n}, 2^{n+k+1}-1\right], F_{n, k}:=$ $\left\{2 p+1: p \in\left[2^{n+k}, 2^{n+k}+2^{n-1}-1\right]\right\}$.

Proof. We first prove that the equality for $\Sigma_{n, k}$ holds for any $n \in \mathbb{N}$ and $k=1$.

$$
\begin{aligned}
A\left(\left\{2^{p}: p \in[1, n+1]\right\} \cup\left\{2^{n}+1\right\}\right) & \\
= & A\left(\left\{2^{p}: p \in[1, n+1]\right\}\right) \cup\left(\left\{2^{n}+1\right\}+A\left(\left\{2^{p}: p \in[1, n+1]\right\}\right)\right) \\
= & \left\{2 p: p \in\left[0,2^{n+1}-1\right]\right\} \cup\left(\left\{2^{n}+1\right\}+\left\{2 p: p \in\left[0,2^{n+1}-1\right]\right\}\right. \\
= & \left\{0,2,4, \ldots, 2^{n}-2,2^{n}, 2^{n}+2, \ldots, 2^{n+2}-4,2^{n+2}-2\right\} \\
\cup & \left\{2^{n}+1,2^{n}+3, \ldots, 2^{n+2}-3,2^{n+2}-1,2^{n+2}\right. \\
& \left.+1, \ldots, 2^{n+2}+2^{n}-3,2^{n+2}+2^{n}-1\right\} \\
= & \left\{0,2,4, \ldots, 2^{n}-2,2^{n}, 2^{n}+1,2^{n}+2, \ldots, 2^{n+2}-2,2^{n+2}\right. \\
& \left.-1,2^{n+2}+1, \ldots, 2^{n+2}+2^{n}-3,2^{n+2}+2^{n}-1\right\} \\
= & \left\{0,2,4, \ldots, 2^{n}-2\right\} \cup\left\{2^{n}, 2^{n}+1,2^{n}+2, \ldots, 2^{n+2}-2,2^{n+2}-1\right\} \\
\cup & \left\{2^{n+2}+1, \ldots, 2^{n+2}+2^{n}-3,2^{n+2}+2^{n}-1\right\} \\
= & B_{n, 1} \cup M_{n, 1} \cup F_{n, 1}
\end{aligned}
$$

Now we assume that the assertion holds for some natural $n$ and $k$. We will prove that it holds for $n$ and $k+1$ which will finish the inductive reasoning.

$$
\begin{aligned}
& A\left(\left\{2^{p}: p \in[1, n+k+1]\right\} \cup\left\{2^{n}+1\right\}\right) \\
&= A\left(\left\{2^{p}: p \in[1, n+k]\right\} \cup\left\{2^{n}+1\right\}\right) \cup\left(\left\{2^{n+k+1}\right\}\right. \\
&\left.+A\left(\left\{2^{p}: p \in[1, n+k]\right\} \cup\left\{2^{n}+1\right\}\right)\right) \\
&=\left\{0,2, \ldots, 2^{n}-2,2^{n}, 2^{n}+1, \ldots, 2^{n+k+1}-2,2^{n+k+1}\right. \\
&\left.-1,2^{n+k+1}+1, \ldots, 2^{n+k+1}+2^{n}-3,2^{n+k+1}+2^{n}-1\right\} \\
& \cup\left\{2^{n+k+1}, \ldots, 2^{n+k+1}+2^{n}-2,2^{n+k+1}+2^{n}, 2^{n+k+1}+2^{n}+1, \ldots,\right. \\
& 2^{n+k+2}-2,2^{n+k+2}-1,2^{n+k+2}+1, \ldots,
\end{aligned}
$$




$$
\begin{aligned}
& \left.2^{n+k+2}+2^{n}-3,2^{n+k+2}+2^{n}-1\right\} \\
= & \left\{0,2, \ldots, 2^{n}-2,2^{n}, 2^{n}+1, \ldots, 2^{n+k+2}-2,2^{n+k+2}\right. \\
& \left.-1,2^{n+k+2}+1, \ldots, 2^{n+k+2}+2^{n}-3,2^{n+k+2}+2^{n}-1\right\} \\
= & \left\{0,2, \ldots, 2^{n}-2\right\} \cup\left\{2^{n}, 2^{n}+1, \ldots, 2^{n+k+2}-2,2^{n+k+2}-1\right\} \\
\cup & \left\{2^{n+k+2}+1, \ldots, 2^{n+k+2}+2^{n}-3,2^{n+k+2}+2^{n}-1\right\} \\
= & B_{n, k+1} \cup M_{n, k+1} \cup F_{n, k+1}
\end{aligned}
$$

Theorem 3.2. For each $\alpha \in(0,1) \cap \mathbb{Q}$ there exists a convergent series $\sum_{i=1}^{\infty} x_{i}$ such that $A\left(x_{i}\right)$ is a Cantorval and the set $\left\{i \in \mathbb{N}: x_{i}>r_{i}\right\}$ has asymptotic density equal to $\alpha$.

Proof. Fix $n, k \in \mathbb{N}$. Let us define the sequence $\left(x_{i}\right)$ as the multigeometric sequence $\left(a_{1}, a_{2}, \ldots, a_{n+k+1} ; q\right)$, that is $x_{(n+k+1) j+p}=a_{p} q^{j}$ for all $j \in \mathbb{N}_{0}$ and $p \in[1, n+k+1]$, where $\left\{a_{1}, a_{2}, \ldots, a_{n+k+1}\right\}=\left\{2^{p}: p \in[1, n+k]\right\} \cup\left\{2^{n}+1\right\}$, that is $a_{j}=2^{n+k+1-j}$ for $j \in[1, k], a_{k+1}=2^{n}+1, a_{j}=2^{n+k+2-j}$ for $j \in$ $[k+2, n+k+1]$. Then by Lemma 3.1 we have $\Sigma=A\left(a_{1}, a_{2}, \ldots, a_{n+k+1}\right)=\Sigma_{n, k}$. Hence, by Theorem 1.3 the achievement set $A\left(a_{1}, a_{2}, \ldots, a_{n+k+1} ; q\right)$ is a Cantorval for each $q \in\left[\frac{1}{2^{n+k+1}-2^{n}}, \frac{2}{2^{n+k+1}+2^{n}+1}\right)$. Clearly $a_{1}>a_{2}>\cdots>$ $a_{n+k+1}$ and $a_{n+k+1}>a_{1} q$ for $q$ in given interval. Thanks to that $\left(x_{n}\right)$ is non-increasing. Note that the role of the set $\left\{n_{0}, n_{0}+1, \ldots, n_{0}+r\right\}$ from Theorem 1.3 is played by the subset $M_{n, k}$ of $\Sigma_{n, k}$. A simple calculation shows that the interval $\left[\frac{1}{2^{n+k+1}-2^{n}}, \frac{2}{2^{n+k+1}+2^{n}+1}\right)$ is nonempty. The condition $q \in$ $\left[\frac{1}{2^{n+k+1}-2^{n}}, \frac{2}{2^{n+k+1}+2^{n}+1}\right)$ means that

$$
\begin{aligned}
& \frac{1}{2^{n+k+1}+2^{n}-1}<\frac{1}{2^{n+k+1}-2^{n}-1}=\frac{\frac{1}{2^{n+k+1}-2^{n}}}{1-\frac{1}{2^{n+k+1}-2^{n}}} \leq \frac{q}{1-q} \\
& \quad<\frac{\frac{2}{2^{n+k+1}+2^{n}+1}}{1-\frac{2}{2^{n+k+1}+2^{n}+1}}=\frac{2}{2^{n+k+1}+2^{n}-1} .
\end{aligned}
$$

Note that

$$
r_{n+k+1}=\sum_{m>n+k+1} x_{m}=\sum_{i=1}^{n+k+1} a_{i} \cdot \frac{q}{1-q}=\left(2^{n+k+2}+2^{n}-1\right) \cdot \frac{q}{1-q} .
$$

Hence we obtain that $r_{n+k+1} \in(1,2)$. Fix $p \in[k+2, k+3, \ldots, n+k+1]$. Then,

$$
\sum_{i=p}^{n+k+1} a_{i}=\sum_{i=p}^{n+k+1} 2^{n+k+2-i}=2^{n+k+3-p}-2
$$


Thus if $p \neq k+2$, then

$$
a_{p-1}-\sum_{i=p}^{n+k+1} a_{i}=2^{n+k+3-p}-\left(2^{n+k+3-p}-2\right)=2>r_{n+k+1}
$$

and also $a_{n+k+1}=2>r_{n+k+1}$. Note that

$$
r_{k+1}=r_{n+k+1}+\sum_{i=k+2}^{n+k+1} a_{i}>1+\left(2^{n+1}-2\right) \geq 2^{n}+1=a_{k+1}
$$

Moreover $\sum_{i=k+1}^{n+k+1} a_{i}=\sum_{i=k+2}^{n+k+1}\left(2^{n+k+2-i}\right)+2^{n}+1=2^{n+1}+2^{n}-1$. Now let $p \in[1, k]$. We have

$$
\begin{gathered}
\sum_{i=p}^{n+k+1} a_{i}=\sum_{i=k+1}^{n+k+1} a_{i}+\sum_{i=p}^{k} a_{i}=2^{n+1}+2^{n}-1+\sum_{i=p}^{k} 2^{n+k+1-i} \\
=2^{n+1}+2^{n}-1+2^{n+k+2-p}-2^{n+1}=2^{n+k+2-p}+2^{n}-1
\end{gathered}
$$

which means that for all $p \in[1, k]$ we have

$$
r_{p}=\sum_{i=p+1}^{n+k+1} a_{i}+r_{n+k+1}>2^{n+k+1-p}+2^{n}>2^{n+k+1-p}=a_{p}
$$

We proved that $\left\{p \in[1, n+k+1]: a_{p}>r_{p}\right\}=[k+2, n+k+1]$. Since we consider the multigeometric sequence $\left(a_{1}, a_{2}, \ldots, a_{n+k+1} ; q\right)$ the following two inequalities are equivalent for all $w \in$ $\mathbb{N}$ and $p \in[1, n+k+1]$ :

$$
a_{(n+k+1) w+p}>r_{(n+k+1) w+p} \Leftrightarrow a_{p}>r_{p}
$$

because $a_{(n+k+1) w+p}=a_{p} \cdot q^{w}$ and $r_{(n+k+1) w+p}=r_{p} \cdot q^{w}$ Then $\left\{i \in \mathbb{N}: x_{i}>\right.$ $\left.r_{i}\right\}=\left\{a(n+k+1)+j: a \in \mathbb{N}_{0}, j \in[k+2, n+k+1]\right\}$, which has asymptotic density $\frac{n}{n+k+1}$. The equality $\left\{\frac{n}{n+k+1}: n, k \in \mathbb{N}\right\}=(0,1) \cap \mathbb{Q}$ is clear.

In order to prove the generalization of the thesis contained in Theorem 3.2 for irrational densities we construct less regular sequences. The idea stays behind the problem of recovering the sequence $\left(x_{i}\right)$ from its achievement set $A\left(x_{i}\right)$. More precisely, it may happen that $A\left(x_{i}\right)=A\left(y_{i}\right)$ for different sequences $\left(x_{i}\right)$ and $\left(y_{i}\right)$ although $A\left(x_{i}\right)$ is obtained as an achievement set uniquely. This problem was deeply studied in [2]. However, we use only a simple observation that $A\left(2^{n}, 2^{n-1}, \ldots, 2\right)=A(\underbrace{2, \ldots, 2}_{2^{n}-1-\text { times }})$.

Remark 3.3. Fix $n, k \in \mathbb{N}$. Let us define the sequence $\left(y_{i}\right)$ as the multigeometric sequence $\left(b_{1}, b_{2}, \ldots, b_{2^{n}+k} ; q\right)$, where $b_{j}=2^{n+k+1-j}$ for $j \in[1, k]$, $b_{k+1}=2^{n}+1, b_{j}=2$ for $j \in\left[k+2,2^{n}+k\right]$. Hence, $\Sigma=A\left(b_{1}, b_{2}, \ldots, b_{2^{n}+k}\right)=$ $\Sigma_{n, k}$, so the achievement set $A\left(b_{1}, b_{2}, \ldots, b_{2^{n}+k} ; q\right)$ is equal to its counterpart $A\left(a_{1}, a_{2}, \ldots, a_{n+k+1} ; q\right)$ constructed in Lemma 3.1. The crucial observation is 
that for the sequence $\left(y_{i}\right)$ the fast convergence condition is satisfied on much thinner set. Indeed, at first, in a very similar reasoning to that in Theorem 3.2 we obtain that $r_{2^{n}+k} \in(1,2)$. Then clearly $b_{2^{n}+k}=2>r_{2^{n}+k}$. Furthermore by dividing the subsequence $a_{k+2}, a_{k+3}, \ldots, a_{n+k+1}$ into constant pieces $b_{k+2}, b_{k+3}, \ldots, b_{2^{n}+k}$ each equal to 2 we break the quick convergence condition obtained on the considered indices without changing the achievement set, that is

$$
A\left(a_{k+2}, a_{k+3}, \ldots, a_{n+k+1}\right)=A\left(b_{k+2}, b_{k+3}, \ldots, b_{2^{n}+k}\right)=\left\{0,2, \ldots, 2^{n+1}-2\right\}
$$

By the same idea as in Theorem 3.2 of shifting computations to the latter indices for multigeometric sequence, we finally get $\left\{i \in \mathbb{N}: y_{i}>r_{i}\right\}=$ $\left\{a\left(2^{n}+k\right): a \in \mathbb{N}\right\}$, which has asymptotic density $\frac{1}{2^{n}+k}$.

By a segment in a multigeometric sequence $\left(a_{1}, a_{2}, \ldots, a_{m} ; q\right)$ we understand the sequence of all the consequitive terms, which are multiplied by the same power of $q$, that is $\left(a_{1} q, a_{2} q, \ldots, a_{m} q\right)$ is the first segment, $\left(a_{1} q^{2}, a_{2} q^{2}, \ldots\right.$, $\left.a_{m} q^{2}\right)$ is the second segment and so on. In the next construction we define the sequence $\left(z_{i}\right)$ segment by segment in the way that each segment is a segment of a multigeometric sequence $\left(x_{i}\right)$ as in the family considered in Theorem 3.2 either its counterpart $\left(y_{i}\right)$ defined in Remark 3.3. Hence, the achievement set $A\left(z_{i}\right)$ will be equal to both $A\left(x_{i}\right)$ and $A\left(y_{i}\right)$. The amounts of both type of segments will depend on the irrational asymptotic density we want to obtain for the set $\left\{i \in \mathbb{N}: z_{i}>r_{i}\right\}$. We will use the following simple result connected with the mediant of two fractions. We give it without proof, since it is basic.

Lemma 3.4. Let $c_{1}, c_{2}, d_{1}, d_{2}$ be natural numbers such that $\frac{c_{1}}{d_{1}}<\frac{c_{2}}{d_{2}}$. Then the function $f:[0,1] \rightarrow \mathbb{R}$ defined as $f(a)=\frac{a c_{2}+(1-a) c_{1}}{a d_{2}+(1-a) d_{1}}$ is continuous and strictly increasing. In particular the range of $f$ is equal to $\left[\frac{c_{1}}{d_{1}}, \frac{c_{2}}{d_{2}}\right]$.

The value $f\left(\frac{1}{2}\right)$ is also known as the mediant of the fractions $\frac{c_{1}}{d_{1}}$ and $\frac{c_{2}}{d_{2}}$.

Theorem 3.5. For each $\alpha \in(0,1)$ there exists a convergent series $\sum_{i=1}^{\infty} z_{i}$ such that $A\left(z_{i}\right)$ is a Cantorval and the set $\left\{i \in \mathbb{N}: z_{i}>r_{i}\right\}$ has asymptotic density equal to $\alpha$.

Proof. Fix $\alpha \in(0,1)$. Let $n$ be any large enough positive integer such that $\alpha \in\left(\frac{1}{2^{n}+1}, \frac{n}{n+2}\right)$. Fix any $q \in\left[\frac{1}{2^{n+2}-2^{n}}, \frac{2}{2^{n+2}+2^{n}+1}\right)$ Define $\left(x_{i}\right)=\left(a_{1}, a_{2}, \ldots\right.$, $\left.a_{n+2} ; q\right)$, where $a_{1}=2^{n+1}, a_{2}=2^{n}+1, a_{j}=2^{n+3-j}$ for $j \in[3, n+2]$ and $\left(y_{i}\right)=\left(b_{1}, b_{2}, \ldots, b_{2^{n}+1} ; q\right)$, where $b_{1}=2^{n+1}, b_{2}=2^{n}+1$ and $b_{j}=2$ for $j \in\left[3,2^{n}+1\right]$.

We construct $\left(z_{i}\right)$ in such a way that the consequitive segments of $\left(z_{i}\right)$ are taken as the segments of $\left(x_{i}\right)$ either $\left(y_{i}\right)$.

Note that if we define first $c+d$ segments of $\left(z_{i}\right)$ by taking $c$ and $d$ segments of $\left(x_{i}\right)$ and $\left(y_{i}\right)$, respectively, then we define $c(n+2)+d\left(2^{n}+1\right)$ 
elements. It also means that $\left|\left\{i \in\left[1, c(n+2)+d\left(2^{n}+1\right)\right]: z_{i}>r_{i}\right\}\right|=c n+d$. By Lemma 3.4 there exists a unique $a \in(0,1)$ such that $\frac{a n+(1-a)}{a(n+2)+(1-a)\left(2^{n}+1\right)}=\alpha$. The value $a$ can be interpreted as the frequency of segments taken from $\left(x_{i}\right)$ in all the segments of the construction of $\left(z_{i}\right)$. If $a \in \mathbb{Q}, a=\frac{u}{w}$, then in the construction of $\left(z_{i}\right)$ we take $u$ segments from $\left(x_{i}\right)$ and then $w-u$ segments from $\left(y_{i}\right)$ and continue in this way. Let us consider the case when $a \in(0,1) \backslash \mathbb{Q}$. Then $a=\sum_{m=1}^{\infty} \frac{\delta_{m}}{10^{m}}$ for some $\left(\delta_{m}\right) \in\{0,1, \ldots, 9\}^{\mathbb{N}}$, which is a decimal expansion of $a=0, \delta_{1} \delta_{2} \ldots$ We consider $a_{p}=\sum_{m=1}^{p} \frac{\delta_{m}}{10^{m}}$, which is a lower rational approximation of $a$ to the $p$-th place after comma, obtained by a simple cut off the expansion in the next places.

We construct $\left(z_{i}\right)$ by taking care about two things. First of them is to cover the proper amounts:

- In the first 10 segments of $\left(z_{i}\right)$ we take exactly $\delta_{1}$ segments from $\left(x_{i}\right)$ and $10-\delta_{1}$ segments from $\left(y_{i}\right)$.

- We add the segments in the way in the first 100 segments of $\left(z_{i}\right)$ there should be exactly $10 \delta_{1}+\delta_{2}$ segments from $\left(x_{i}\right)$ and $100-10 \delta_{1}-\delta_{2}$ segments from $\left(y_{i}\right)$.

- We continue inductively and obtain that for each $t \in \mathbb{N}$ the first $10^{t}$ segments of $\left(z_{i}\right)$ contain exactly $\sum_{j=1}^{t} \delta_{j} 10^{t-j}$ segments from $\left(x_{i}\right)$ and the remaining ones are from $\left(y_{i}\right)$.

The second condition is the order of segments:

When we define the $k$-th segment, $k \in[1,10]$ for $t=1$ either $k \in\left[10^{t-1}+1,10^{t}\right]$ for some $t \in \mathbb{N}, t \geq 2$, then we consider the ratio $\beta_{k-1}$ of number of segments $\left(x_{i}\right)$ contained in the first $k-1$ segments of $\left(z_{i}\right)$ to $k-1$. We also put $\beta_{0}=0$. If $\beta_{k-1} \leq \sum_{m=1}^{t} \frac{\delta_{m}}{10^{m}}$, then as the $k$-th segment we take (if we can) the $k$-th segment of $\left(x_{i}\right)$, otherwise we take the $k$-th segment of $\left(y_{i}\right)$. The , if we can" part is connected with the situation, when $a$ is such small that $\delta_{m}=0$ for all $m \leq t$. Then, by the first condition of construction, all of first $10^{t}$ segments of $\left(z_{i}\right)$ are taken from $\left(y_{i}\right)$.

From the construction we obtain that for each $t$ the cardinality of the set $\left\{i \in\left[1, \sum_{j=1}^{t} \delta_{j} 10^{t-j}(n+2)+\left(10^{t}-\sum_{j=1}^{t} \delta_{j} 10^{t-j}\right)\left(2^{n}+1\right)\right]: z_{i}>r_{i}\right\}$ equals $n \sum_{j=1}^{t} \delta_{j} 10^{t-j}+10^{t}-\sum_{j=1}^{t} \delta_{j} 10^{t-j}$. Note that $\frac{n \sum_{j=1}^{t} \delta_{j} 10^{t-j}+10^{t}-\sum_{j=1}^{t} \delta_{j} 10^{t-j}}{\sum_{j=1}^{t} \delta_{j} 10^{t-j}(n+2)+\left(10^{t}-\sum_{j=1}^{t} \delta_{j} 10^{t-j}\right)\left(2^{n}+1\right)}$ tends to $\alpha$ as $t$ tends to $\infty$. The second condition of the construction guarantees alternating-like convergence of $\beta_{k}$ to $a$. Clearly, $\beta_{10^{t}}=\sum_{m=1}^{t} \frac{\delta_{m}}{10^{m}}$ for each $t \in \mathbb{N}$. Assume that $t \geq \min \left\{t_{0}: \delta_{t_{0}} \neq\right.$ $0\}$. Since $\sum_{m=1}^{t} \frac{\delta_{m}}{10^{m}} \leq \sum_{m=1}^{t+1} \frac{\delta_{m}}{10^{m}}$ by the second rule of the construction, as the $10^{t}+1$-th segment we always take $\left(x_{i}\right)$.

We take segments of $\left(x_{i}\right)$ until $\left(\beta_{k}\right)$ exceeds the value of $\beta_{10^{t+1}}$. Then, we continue with taking $\left(y_{i}\right)$ to the moment we get values of $\left(\beta_{k}\right)$ not greater than $\beta_{10^{t+1}}$ and so on. Since the number of the defined elements tends to infinity, 
the affection to the ratio $\left(\beta_{k}\right)$ by the consequitve $n+2$ either $2^{n}+1$ elements is not significant and tends to zero.

For the constructed series $\sum_{i=1}^{\infty} z_{i}$ we have that $A\left(z_{i}\right)=A\left(x_{i}\right)=A\left(y_{i}\right)$, so it is a Cantorval and the set $\left\{i \in \mathbb{N}: z_{i}>r_{i}\right\}$ has asymptotic density equal to $\alpha$.

Remark 3.6. Note that if $\delta_{m}=9$ for $m \leq t+1$, then all of the segments between $10^{t}+1$ and $10^{t+1}$ will be taken from $\left(x_{i}\right)$. However, it does not affect the convergence of $\left(\beta_{k}\right)$, because the sequence is increasing in that indices. Unfortunately, we are not able to use the similar method of construction to obtain a Cantorval for which the set $\left\{i \in \mathbb{N}: z_{i}>r_{i}\right\}$ has asymptotic density equal to 1 . The proof breaks on the beginning, when we are looking for $n$ such that $\frac{n}{n+2}$ is greater than the considered density.

Theorem 3.7. For each $0<\alpha \leq \beta<1$ there exists a convergent series $\sum_{i=1}^{\infty} z_{i}$ such that $A\left(z_{i}\right)$ is a Cantorval and the set $\left\{i \in \mathbb{N}: z_{i}>r_{i}\right\}$ has lower asymptotic density equal to $\alpha$ and upper asymptotic density equal to $\beta$.

Proof. The case $\alpha=\beta$ has been already proved in Theorem 3.5. Let $n$ be any large enough positive integer such that $\frac{1}{2^{n}+1}<\alpha<\beta<\frac{n}{n+2}$. The sequences $\left(x_{i}\right),\left(y_{i}\right)$ and the value of $q$ are defined in the same way as in Theorem 3.5. We define $\left(z_{i}\right)$ by using the segments of $\left(x_{i}\right)$ either $\left(y_{i}\right)$ in the following way:

- The first segment is a segment of $\left(x_{i}\right)$.

- Then, we add the least number of segments $\left(y_{i}\right)$ as is needed to obtain the fast convergence condition on less than $\alpha$ of the defined terms since the beginning of the construction. The sequence of all the defined terms in this step will be also called as a block of segments of $\left(y_{i}\right)$.

- After that we take the least number of segments $\left(x_{i}\right)$ that we need to get the fast convergence condition on more then $\beta$ of the defined terms since the beginning of the construction, that is a block of segments of $\left(x_{i}\right)$.

- We continue inductively.

Let $u_{j}$ be the number of all defined terms of $\left(z_{i}\right)$ after taking $j$ first blocks of segments of $\left(y_{i}\right)$ and $j-1$ first blocks of segments of and $\left(x_{i}\right)$, respectively. Similarly, let $w_{j}$ be the number of all defined terms of $\left(z_{i}\right)$ after taking $j$ first blocks of segments of $\left(y_{i}\right)$ and $j$ first blocks of segments of and $\left(x_{i}\right)$, respectively. Clearly, $\lim _{j \rightarrow \infty} \frac{\left|\left\{i \in\left[1, u_{j}\right]: z_{i}>r_{i}\right\}\right|}{u_{j}}=\alpha$ and $\lim _{j \rightarrow \infty} \frac{\left|\left\{i \in\left[1, w_{j}\right]: z_{i}>r_{i}\right\}\right|}{w_{j}}=\beta$. From the construction we see that $\alpha$ and $\beta$ are lower and upper asymptotic density of the set $\left\{i \in \mathbb{N}: z_{i}>r_{i}\right\}$, respectively.

Remark 3.8. The proof of Theorem 3.5 can be rewritten to use the more similar method to the way of construction in Theorem 3.7. Namely, instead of comparing the sequence $\left(\beta_{k}\right)$ with consequtive $a_{p}$ 's, we may immediately use the value of $a$. It causes many advantages like simplifying the proof, since the notion of $\left(\delta_{m}\right)$ is not needed. Hence, we never change the value with which we compare the terms of $\left(\beta_{k}\right)$ with. The convergence of $\left(\beta_{k}\right)$ to $a$ is also faster 
then. Another change is in a slight increase of the chosen segments from $\left(x_{i}\right)$ in the beginning of the construction. However, comparing to the irrational value of $a$ may lose the intuition in the sense we only know that the frequency $\left(\beta_{k}\right)$ of taking segments from $\left(x_{i}\right)$ oscilates close to $a$. It is impossible to predict the terms of $\left(\beta_{k}\right)$ accurately without calculating the previous terms. In the original proof of Theorem 3.5 we know what we obtain for $\beta_{10^{t}}$ for each $t \in \mathbb{N}$ on the spot what determines the amount of taken segments from each type.

Theorem 3.9. There exists a convergent series $\sum_{i=1}^{\infty} z_{i}$ such that $A\left(z_{i}\right)$ is a Cantorval and the set $\left\{i \in \mathbb{N}: z_{i}>r_{i}\right\}$ has asymptotic density equal to 0 .

Proof. Let $G N=A\left(x_{i}\right)=A\left(3,2 ; \frac{1}{4}\right)$ be the Guthrie and Nymann's Cantorval. We consider its superset $A\left(y_{i}\right)=A\left(1,1,1,1,1 ; \frac{1}{4}\right)=\left[0, \frac{5}{3}\right]$. We construct a sequence $\left(z_{i}\right)$ again by taking segments from $\left(x_{i}\right)$ either $\left(y_{i}\right)$. In the first step we take 5 segments from $\left(x_{i}\right)$ and after that 18 segments from $\left(y_{i}\right)$. For each $k \geq 2, k \in \mathbb{N}$, in the $k$-th step we define 5 segments from $\left(x_{i}\right)$ and then $18 \cdot 10^{k-1}-2$ segments from $\left(y_{i}\right)$. It means that in the first step we define 100 terms and in the $k$-th step for $k \geq 2$ we define exactly $9 \cdot 10^{k}$ consequitive elements of $\left(z_{i}\right)$. Hence for any $k \in \mathbb{N}$ in all of the first $k$-th steps we define $10^{k+1}$ terms of $\left(z_{i}\right)$. Note that by the construction we obtain that $\{i \in \mathbb{N}$ : $\left.z_{i}>r_{i}\right\}=\{2,4,6,8,10\} \cup\left\{10^{k+1}+j: k \in \mathbb{N}, j \in\{2,4,6,8,10\}\right\}$. Clearly

$$
\begin{aligned}
& \limsup _{n \rightarrow \infty} \frac{\left|\left\{i \in\{1, \ldots, n\}: z_{i}>r_{i}\right\}\right|}{n}=\lim _{n \rightarrow \infty} \frac{\left|\left\{i \in\left\{1, \ldots, 10^{n+1}+10\right\}: z_{i}>r_{i}\right\}\right|}{10^{n+1}+10} \\
& \quad=\lim _{n \rightarrow \infty} \frac{5 n+5}{10^{n+1}+10}=0
\end{aligned}
$$

Thus the set $\left\{i \in \mathbb{N}: z_{i}>r_{i}\right\}$ has asymptotic density equal to 0 . Since the fast convergence condition is satisfied on infinite set of indices, by Theorem 1.1(1) we know that $A\left(z_{i}\right)$ is not a finite union of closed intervals. However $A\left(z_{i}\right) \supset$ $G N$, which means that $A\left(z_{i}\right)$ is a Cantorval.

By combining the method used in Theorem 3.7 and construction similar to that one in Theorem 3.9 we obtain the following.

Theorem 3.10. For each $0 \leq \beta<1$ there exists a convergent series $\sum_{i=1}^{\infty} z_{i}$ such that $A\left(z_{i}\right)$ is a Cantorval and the set $\left\{i \in \mathbb{N}: z_{i}>r_{i}(\boldsymbol{z})\right\}$ has lower asymptotic density equal to 0 and upper asymptotic density equal to $\beta$.

Proof. It is enough to combine $\left(z_{i}\right)$ from the segments belonging to the sequences $\left(x_{i}\right)$ and $\left(y_{i}\right)$ for which fast convergence condition is satisfied on the set with asymptotic density 0 and $\beta$, respectively. To be more precise, we take any sequence $\left(y_{i}\right)$ such that the series $\sum_{i=1}^{\infty} y_{i}$ is convergent, the set $A\left(y_{i}\right)$ is a Cantorval and the set $\left\{i \in \mathbb{N}: y_{i}>r_{i}(\mathbf{y})\right\}$ has asymptotic density equal to $\beta$ (such a sequence exists by virtue of Theorem 3.5). Then, we define $\left(x_{i}\right)=(\underbrace{1, \ldots, 1}_{k \text { times }} ; q)$, where $q$ is as in the construction of $\left(y_{i}\right)$ and $k \geq \frac{1}{q}$ 
is so large that $A\left(x_{i}\right)=\left[0, \frac{k q}{1-q}\right]$ is a superset of $A\left(y_{i}\right)$. Of course, the fast convergence condition is never satisfied in the case of the sequence $\left(x_{i}\right)$.

In the $2 k-1$-st step, $k \in \mathbb{N}$, we define the least number of segments from $\left(y_{i}\right)$ to obtain the fast convergence condition on more than $\beta-\frac{1}{k}$ of all defined indices.

In the $2 k$-th step, $k \in \mathbb{N}$, we define the least number of segments from $\left(x_{i}\right)$ to obtain the fast convergence condition on less than $\frac{1}{k}$ of all defined indices.

In the end of this section we gather our results in the following corollary.

Corollary 3.11. For each $0 \leq \alpha \leq \beta<1$ there exists a convergent series $\sum_{i=1}^{\infty} z_{i}$ such that $A\left(z_{i}\right)$ is a Cantorval and the set $\left\{i \in \mathbb{N}: z_{i}>r_{i}\right\}$ has lower asymptotic density equal to $\alpha$ and upper asymptotic density equal to $\beta$.

\section{Examples}

In this chapter we present numerical examples of Theorems 3.2, 3.5, 3.7. We start with the simplest consequences of Theorem 3.2.

Example 4.1. The set $A(4,3,2 ; q)$ is a Cantorval for each $q \in\left[\frac{1}{6}, \frac{2}{11}\right)$. The fast convergence condition is satisfied on $3 \mathbb{N}$ with density $\frac{1}{3}$.

Example 4.2. The set $A(32,17,16,8,4,2 ; q)$ is a Cantorval for each $q \in\left[\frac{1}{48}, \frac{2}{81}\right)$. The fast convergence condition is satisfied on $(6 \mathbb{N}-3) \cup(6 \mathbb{N}-2) \cup(6 \mathbb{N}-1) \cup(6 \mathbb{N})$ with density $\frac{2}{3}$.

Now we present the calculations for the first segments of the series for which the fast convergence condition is satisfied on the set with irrational density, see Theorem 3.5.

Example 4.3. We show how to define the beginning segments of a convergent series $\sum_{i=1}^{\infty} z_{i}$ such that $A\left(z_{i}\right)$ is a Cantorval and the set $\left\{i \in \mathbb{N}: z_{i}>r_{i}\right\}$ has an asymptotic density equal to $\alpha=\frac{\pi}{10}=0.314159 \ldots$

For $n=2$ we have $\frac{1}{2^{n}+1}=0.2<\alpha<0.5=\frac{n}{n+2}$. Thus we will take the segments to construct $\left(z_{i}\right)$ from the multigeometric sequence $\left(x_{i}\right)=$ $(8,5,4,2 ; q)$ either $\left(y_{i}\right)=(8,5,2,2,2 ; q)$. We also fix $q$ as any number from the interval $\left[\frac{1}{12}, \frac{2}{21}\right)$.

The equality $\frac{a n+(1-a)}{a(n+2)+(1-a)\left(2^{n}+1\right)}=\alpha$ has a solution $a=0.434 \ldots$ Hence for the first group of segments the choice between $\left(x_{i}\right)$ and $\left(y_{i}\right)$ is determined by $\beta_{10}=0.4$. 


\begin{tabular}{|c|c|c|c|c|}
\hline $\begin{array}{l}\text { No. } \\
\text { of } \\
\text { seg- } \\
\text { ment } \\
k\end{array}$ & $\begin{array}{l}\text { Defined } \\
\text { type }\end{array}$ & $\begin{array}{l}\text { Defined } \\
\text { terms }\end{array}$ & $\beta_{k}$ & $\frac{\text { Number of terms with fast conv. cond. }}{\text { Number of all defined terms }}$ \\
\hline 1 & $\left(x_{i}\right)$ & $\begin{array}{l}8 q, 5 q \\
4 q, 2 q\end{array}$ & 1 & $\frac{2}{4}=0.5$ \\
\hline 2 & $\left(y_{i}\right)$ & $\begin{array}{ll}8 q^{2}, & 5 q^{2} \\
2 q^{2}, & 2 q^{2} \\
2 q^{2} & \end{array}$ & $\frac{1}{2}=0.5$ & $\frac{3}{9} \approx 0.33$ \\
\hline 3 & $\left(y_{i}\right)$ & $\begin{array}{ll}8 q^{3}, & 5 q^{3} \\
2 q^{3}, & 2 q^{3} \\
2 q^{3} & \end{array}$ & $\frac{1}{3} \approx 0.33$ & $\frac{4}{14} \approx 0.29$ \\
\hline 4 & $\left(x_{i}\right)$ & $\begin{array}{l}8 q^{4}, \quad 5 q^{4} \\
4 q^{4}, 2 q^{4}\end{array}$ & $\frac{2}{4}=0.5$ & $\frac{6}{18} \approx 0.33$ \\
\hline 5 & $\left(y_{i}\right)$ & $\begin{array}{ll}8 q^{5}, & 5 q^{5} \\
2 q^{5} & 2 q^{5} \\
2 q^{5} & \end{array}$ & $\frac{2}{5}=0.4$ & $\frac{7}{23} \approx 0.304$ \\
\hline 6 & $\left(x_{i}\right)$ & $\begin{array}{l}8 q^{6}, \quad 5 q^{6} \\
4 q^{6}, 2 q^{6}\end{array}$ & $\frac{3}{6}=0.5$ & $\frac{9}{27} \approx 0.33$ \\
\hline 7 & $\left(y_{i}\right)$ & $\begin{array}{ll}8 q^{7}, & 5 q^{7} \\
2 q^{7}, & 2 q^{7} \\
2 q^{7} & \end{array}$ & $\frac{3}{7} \approx 0.428$ & $\frac{10}{32} \approx 0.3125$ \\
\hline 8 & $\left(y_{i}\right)$ & $\begin{array}{ll}8 q^{8}, & 5 q^{8} \\
2 q^{8}, & 2 q^{8} \\
2 q^{8} & \end{array}$ & $\frac{3}{8}=0.375$ & $\frac{11}{37} \approx 0.297$ \\
\hline 9 & $\left(x_{i}\right)$ & $\begin{array}{l}8 q^{9}, \quad 5 q^{9} \\
4 q^{9}, 2 q^{9}\end{array}$ & $\frac{4}{9} \approx 0.44$ & $\frac{13}{41} \approx 0.317$ \\
\hline 10 & $\left(y_{i}\right)$ & $\begin{array}{l}8 q^{10}, \quad 5 q^{10} \\
2 q^{10}, \\
2 q^{10}\end{array}$ & $\frac{4}{10}=0.4$ & $\frac{14}{46} \approx 0.304$ \\
\hline
\end{tabular}

Now we define the second group of segments. Here we compare the terms $\beta_{k-1}$ with the value $\beta_{100}=0.43$. 


\begin{tabular}{|c|c|c|c|c|}
\hline $\begin{array}{l}\text { No. } \\
\text { of } \\
\text { seg- } \\
\text { ment } \\
k\end{array}$ & $\begin{array}{l}\text { Defined } \\
\text { type }\end{array}$ & $\begin{array}{l}\text { Defined } \\
\text { terms }\end{array}$ & $\beta_{k}$ & $\frac{\text { Number of terms with fast conv. cond }}{\text { Number of all defined terms }}$ \\
\hline 11 & $\left(x_{i}\right)$ & $\begin{array}{l}8 q^{11}, 5 q^{11} \\
4 q^{11}, 2 q^{11}\end{array}$ & $\frac{5}{11} \approx 0.45$ & $\frac{16}{50}=0.32$ \\
\hline 12 & $\left(y_{i}\right)$ & $\begin{array}{l}8 q^{12}, 5 q^{12} \\
2 q^{12}, \\
2 q^{12}\end{array}$ & $\frac{5}{12} \approx 0.42$ & $\frac{17}{55} \approx 0.309$ \\
\hline 13 & $\left(x_{i}\right)$ & $\begin{array}{l}8 q^{13}, 5 q^{13} \\
4 q^{13}, 2 q^{13}\end{array}$ & $\frac{6}{13} \approx 0.46$ & $\frac{19}{59} \approx 0.322$ \\
\hline 14 & $\left(y_{i}\right)$ & $\begin{array}{l}8 q^{14}, 5 q^{14} \\
2 q^{14}, 2 q^{14} \\
2 q^{14}\end{array}$ & $\frac{6}{14} \approx 0.429$ & $\frac{20}{64} \approx 0.3125$ \\
\hline 15 & $\left(x_{i}\right)$ & $\begin{array}{l}8 q^{15}, 5 q^{15} \\
4 q^{15}, 2 q^{15}\end{array}$ & $\frac{7}{15} \approx 0.47$ & $\frac{22}{68} \approx 0.324$ \\
\hline 16 & $\left(y_{i}\right)$ & $\begin{array}{l}8 q^{16}, 5 q^{16} \\
2 q^{16}, \\
2 q^{16}\end{array}$ & $\frac{7}{16} \approx 0.438$ & $\frac{23}{73} \approx 0.315$ \\
\hline 17 & $\left(y_{i}\right)$ & $\begin{array}{l}8 q^{17}, 5 q^{17} \\
2 q^{17}, \\
2 q^{17}\end{array}$ & $\frac{7}{17} \approx 0.41$ & $\frac{24}{78} \approx 0.308$ \\
\hline 18 & $\left(x_{i}\right)$ & $\begin{array}{l}8 q^{18}, 5 q^{18} \\
4 q^{18}, 2 q^{18}\end{array}$ & $\frac{8}{18} \approx 0.44$ & $\frac{26}{82} \approx 0.317$ \\
\hline$\vdots$ & $\vdots$ & $\vdots$ & $\vdots$ & $\cdot$ \\
\hline 100 & $\ldots$ & $\ldots$ & $\frac{43}{100}=0.43$ & $\frac{143}{457} \approx 0.31291$ \\
\hline
\end{tabular}

In the third group we consider $\beta_{1000}=0.434$.

\begin{tabular}{lllll}
\hline $\begin{array}{l}\text { No. } \\
\text { of } \\
\text { seg- } \\
\text { ment }\end{array}$ & $\begin{array}{l}\text { Defined } \\
\text { type }\end{array}$ & $\begin{array}{l}\text { Defined } \\
\text { terms }\end{array}$ & $\beta_{k}$ & $\frac{\text { Number of terms with fast conv. cond. }}{\text { Number of all defined terms }}$ \\
$k$ & & $\vdots$ & $\vdots$ & $\vdots$ \\
\hline$\vdots$ & $\vdots$ & $\vdots$ & $\frac{434}{1000}=0.434$ & $\frac{1434}{4566} \approx 0.31406$ \\
1000 & $\ldots$ & $\ldots$ & & \\
\hline
\end{tabular}

and so on. By the construction $A\left(z_{i}\right)=A\left(x_{i}\right)=A\left(y_{i}\right)$ is a Cantorval. 
Remark 4.4. Let us consider again Example 4.3. Due to Remark 3.8 in the modified method the first change appears in the eight segment. Since $\beta_{7}<a$ the segment in the eight step is taken from $\left(x_{i}\right)$. It may also affect the segments defined in the next steps.

In the next Example we show how to construct a convergent series $\sum_{i=1}^{\infty} z_{i}$ such that $A\left(z_{i}\right)$ is a Cantorval and the set $\left\{i \in \mathbb{N}: z_{i}>r_{i}\right\}$ has a given lower and upper assymptotic densities as in the Theorem 3.7.

Example 4.5. We define a convergent series $\sum_{i=1}^{\infty} z_{i}$ such that $A\left(z_{i}\right)$ is a Cantorval and the set $\left\{i \in \mathbb{N}: z_{i}>r_{i}\right\}$ has a lower asymptotic density equal to $\alpha=0.15$ and upper asymptotic density equal to $\beta=0.55$.

For $n=3$ we have $\frac{1}{2^{n}+1}<\alpha<\beta<\frac{n}{n+2}$. Thus we will take the segments to construct $\left(z_{i}\right)$ from the multigeometric sequence $\left(x_{i}\right)=(16,9,8,4,2 ; q)$ either $\left(y_{i}\right)=(16,9,2,2,2,2,2,2,2 ; q)$. We also fix $q$ as any number from the interval $\left[\frac{1}{24}, \frac{2}{41}\right)$.

\begin{tabular}{|c|c|c|c|}
\hline $\begin{array}{l}\text { No. of } \\
\text { segment } \\
k\end{array}$ & $\begin{array}{l}\text { Defined } \\
\text { type }\end{array}$ & $\begin{array}{l}\text { Defined } \\
\text { terms }\end{array}$ & $\frac{\text { Number of terms with fast conv. cond. }}{\text { Number of all defined terms }}$ \\
\hline 1 & $\left(x_{i}\right)$ & $16 q 9 q, 8 q, 4 q, 2 q$ & $\frac{3}{5}=0.6$ \\
\hline 2 & $\left(y_{i}\right)$ & $16 q^{2}, 9 q^{2}, 2 q^{2}, \ldots, 2 q^{2}$ & $\frac{4}{14} \approx 0.29$ \\
\hline 3 & $\left(y_{i}\right)$ & $16 q^{3}, 9 q^{3}, 2 q^{3}, \ldots, 2 q^{3}$ & $\frac{\frac{15}{23}}{23} \approx 0.22$ \\
\hline 4 & $\left(y_{i}\right)$ & $16 q^{4}, 9 q^{4}, 2 q^{4}, \ldots, 2 q^{4}$ & $\frac{6}{32} \approx 0.19$ \\
\hline 5 & $\left(y_{i}\right)$ & $16 q^{5}, 9 q^{5}, 2 q^{5}, \ldots, 2 q^{5}$ & $\frac{7}{41} \approx 0.17$ \\
\hline 6 & $\left(y_{i}\right)$ & $16 q^{6}, 9 q^{6}, 2 q^{6}, \ldots, 2 q^{6}$ & $\frac{\frac{4}{8}}{50}=0.16$ \\
\hline 7 & $\left(y_{i}\right)$ & $16 q^{7}, 9 q^{7}, 2 q^{7}, \ldots, 2 q^{7}$ & $\frac{9}{59} \approx 0.153$ \\
\hline 8 & $\left(y_{i}\right)$ & $16 q^{8}, 9 q^{8}, 2 q^{8}, \ldots, 2 q^{8}$ & $\frac{10}{68} \approx 0.147$ \\
\hline 9 & $\left(x_{i}\right)$ & $16 q^{9}, 9 q^{9}, 8 q^{9}, 4 q^{9}, 2 q^{9}$ & $\frac{13}{73} \approx 0.178$ \\
\hline$\vdots$ & $\vdots$ & $\vdots$ & $\vdots$ \\
\hline 117 & $\left(x_{i}\right)$ & $\begin{array}{ll}16 q^{117}, & 9 q^{117} \\
8 q^{117}, & 4 q^{117} \\
2 q^{117} & \end{array}$ & $\frac{337}{613} \approx 0.5498$ \\
\hline 118 & $\left(x_{i}\right)$ & $\begin{array}{ll}16 q^{118}, & 9 q^{118} \\
8 q^{118}, & 4 q^{118} \\
2 q^{118} & \end{array}$ & $\frac{340}{618} \approx 0.5502$ \\
\hline 119 & $\left(y_{i}\right)$ & $\begin{array}{l}16 q^{119}, \quad 9 q^{119} \\
2 q^{119}, \ldots, 2 q^{119}\end{array}$ & $\frac{341}{627} \approx 0.544$ \\
\hline$\vdots$ & $\vdots$ & $\vdots$ & $\vdots$ \\
\hline
\end{tabular}




\begin{tabular}{|c|c|c|c|}
\hline $\begin{array}{l}\text { No. of } \\
\text { segment } \\
k\end{array}$ & $\begin{array}{l}\text { Defined } \\
\text { type }\end{array}$ & $\begin{array}{l}\text { Defined } \\
\text { terms }\end{array}$ & $\frac{\text { Number of terms with fast conv. cond. }}{\text { Number of all defined terms }}$ \\
\hline 824 & $\left(y_{i}\right)$ & $\begin{array}{l}16 q^{824}, \quad 9 q^{824} \\
2 q^{824}, \ldots, 2 q^{824}\end{array}$ & $\frac{1046}{6972} \approx 0.15003$ \\
\hline 825 & $\left(y_{i}\right)$ & $\begin{array}{l}16 q^{825}, \quad 9 q^{825} \\
2 q^{825}, \ldots, 2 q^{825}\end{array}$ & $\frac{1047}{6981} \approx 0.14998$ \\
\hline 826 & $\left(x_{i}\right)$ & $\begin{array}{ll}16 q^{826}, & 9 q^{826}, \\
8 q^{826}, & 4 q^{826} \\
2 q^{826} & \end{array}$ & $\frac{1050}{6986} \approx 0.1503$ \\
\hline$\vdots$ & $\vdots$ & $\vdots$ & $\vdots$ \\
\hline
\end{tabular}

\section{Open Problems}

In the last chapter we show some of the possible ways of continuation for the study of connections between Kakeya conditions and topology of achievment sets. We start with the most general question that we suppose that its answer is affirmative.

Problem 5.1. Is it possible for each infinite subset $C$ of $\mathbb{N}$ with infinite complement to construct a convergent series $\sum_{i=1}^{\infty} z_{i}$ such that $A\left(z_{i}\right)$ is a Cantorval and $\left\{i \in \mathbb{N}: z_{i}>r_{i}\right\}=C$ ?

We expect that the above problem can be very difficult to attack. This is why we propose to start with a particular case.

Problem 5.2. Is it possible to construct a convergent series $\sum_{i=1}^{\infty} z_{i}$ such that $A\left(z_{i}\right)$ is a Cantorval and the set $\left\{i \in \mathbb{N}: z_{i}>r_{i}\right\}$ has asymptotic density equal to 1 ?

Note that in the paper we have shown that such Examples exist for all densities $\alpha \in[0,1)$. However, if the answer is true the construction may need completely different methods than used in the paper. On the other hand, if the answer for the above question is negative we may also ask the following.

Problem 5.3. Is it possible to construct a convergent series $\sum_{i=1}^{\infty} z_{i}$ such that $A\left(z_{i}\right)$ is a Cantorval and the set $\left\{i \in \mathbb{N}: z_{i}>r_{i}\right\}$ has upper asymptotic density equal to 1 ?

In the paper we measure the size of the set $\left\{i \in \mathbb{N}: z_{i}>r_{i}\right\}$ by the notion of asymptotic density. However, one can study its structure much deeper. Namely, we say that the set $E \subset \mathbb{N}$ belongs to the interval class $\mathcal{I}$ (Cantor class $\mathcal{C}$ or Cantorval class $\mathcal{C} \mathcal{V})$ if there exists a convergent series $\sum_{i=1}^{\infty} z_{i}$ such that $A\left(z_{i}\right)$ is a finite union of closed intervals (homemorphic to a Cantor set or Cantorval, respectively) and $E=\left\{i \in \mathbb{N}: z_{i}>r_{i}\right\}$. By Theorem 1.1(1) 
it is obvious that $\mathcal{I}$ is equal to the family of all finite subsets of $\mathbb{N}$, that is $\mathcal{I}=$ Fin and $\mathcal{I} \cap(\mathcal{C} \cup \mathcal{C V})=\emptyset$. Furthermore, by Theorem 2.1 we know that $\mathcal{C}=P(\mathbb{N}) \backslash$ Fin. Hence, we may state the following problem.

Problem 5.4. Characterize the family $\mathcal{C} \mathcal{V}$.

Clearly, $(\mathcal{C} \cap \mathcal{C V}) \neq \emptyset$. Another simple observation is that removing or adding finitely many terms to the sequence $\left(z_{i}\right)$ does not change the form of its achievement set, so if some $A \in \mathcal{C} \mathcal{V}$, then each set of the form $A \Delta F$ for some $F \in$ Fin is an element of $\mathcal{C} \mathcal{V}$ as well. In particular, by Theorem 3.2 we obtain that $a \mathbb{N}+B \in \mathcal{C} \mathcal{V}$ for each $a \in \mathbb{N}$ and $B$, which is any subset of $\{1, \ldots, a\}$ containing consecutive numbers. Also, by Theorem 1.1(2) we know that if $C \subset \mathbb{N}$ has finite complement, then $C \in \mathcal{C} \backslash \mathcal{C V}$.

\section{Acknowledgements}

We are grateful to an anonymous referee for their careful reading and remarks that helped improving the edition of the paper.

Funding The research of the second author is supported by the Grant of the Polish National Science Centre No. UMO-2019/34/E/ST1/00094.

\section{Declarations}

Conflict of interest There are neither conflicts of interest nor competing interests.

Open Access. This article is licensed under a Creative Commons Attribution 4.0 International License, which permits use, sharing, adaptation, distribution and reproduction in any medium or format, as long as you give appropriate credit to the original author(s) and the source, provide a link to the Creative Commons licence, and indicate if changes were made. The images or other third party material in this article are included in the article's Creative Commons licence, unless indicated otherwise in a credit line to the material. If material is not included in the article's Creative Commons licence and your intended use is not permitted by statutory regulation or exceeds the permitted use, you will need to obtain permission directly from the copyright holder. To view a copy of this licence, visit http://creativecommons. org/licenses/by/4.0/.

\section{References}

[1] Banakh, T., Bartoszewicz, A., Filipczak, M., Szymonik, E.: Topological and measure properties of some self-similar sets. Topol. Methods Nonlinear Anal. 46(2), 1013-1028 (2015)

[2] Bartoszewicz, A., Głąb, S., Marchwicki, J. : Recovering a purely atomic finite measure from its range. J. Math. Anal. Appl. 467, 825-841 (2018) 
[3] Banakh, T., Bartoszewicz, A., Głąb, S., Szymonik, E.: Algebraic and topological properties of some sets in $\ell_{1}$, Colloq. Math. 129, 75-85 (2012)

[4] Bartoszewicz, A., Filipczak, M., Prus-Wiśniowski, F.: Topological and Algebraic Aspects of Subsums of Series, Traditional and Present-Day Topics in Real Analysis, pp. 345-366. Faculty of Mathematics and Computer Science, University of Łódź, Łódź (2013)

[5] Bartoszewicz, A., Filipczak, M., Szymonik, E.: Multigeometric sequences and Cantorvals. Cent. Eur. J. Math. 12(7), 1000-1007 (2014)

[6] Daróczy, Z., Járai, A., Katái, I.: Intervallfullende Folgen und volladditive Funktionen. Acta Sci. Math. 50, 337-350 (1986)

[7] Daróczy, Z., Katái, I.: Interval filling and additive functions. Acta Sci. Math. 52, 337-347 (1988)

[8] Daróczy, Z., Katái, I., Szabó, T.: On completely additive functions related to interval-filling sequences. Arch. Math. 54, 173-179 (1990)

[9] Ferens, C.: On the range of purely atomic probability measures. Stud. Math. 77(3), 261-263 (1984)

[10] Głąb, S., Marchwicki, J.: Cardinal functions of atomic measures. Results Math.75(4), Article number: 141 (2020), 26 pp

[11] Guthrie, J.A., Nymann, J.E.: The topological structure of the set of subsums of an infinite series. Colloq. Math. 55(2), 323-327 (1988)

[12] Kakeya, S.: On the partial sums of an infinite series. Tôhoku Sic. Rep. 3, 159-164 (1914)

[13] Mendes, P., Oliveira, F.: On the topological structure of the arithmetic sum of two Cantor sets. Nonlinearity 7, 329-343 (1994)

[14] Nymann, J.E., Sáenz, R.A.: On the paper of Guthrie and Nymann on subsums of infinite series. Colloq. Math. 83, 1-4 (2000)

[15] Prus-Wiśniowski, F.: Beyond the sets of subsums. Preprints of the Faculty of Mathematics and Informatics, Lodz University (2013)

[16] Weinstein, A.D., Shapiro, B.E.: On the structure of a set of $\bar{\alpha}$-representable numbers. Izv. Vysš. Učebn. Zaved. Mat. 24, 8-11 (1980)

Jacek Marchwicki

Chair of Complex Analysis, Faculty of Mathematics and Computer Science

University of Warmia and Mazury in Olsztyn

Słoneczna 54

10-710 Olsztyn

Poland

e-mail: marchewajaclaw@gmail .com 
Piotr Miska

Faculty of Mathematics and Computer Science, Institute of Mathematics

Jagiellonian University in Kraków

ul. Łojasiewicza 6

30-348 Kraków

Poland

e-mail: piotr.miska@uj.edu.pl

Received: March 6, 2021.

Accepted: July 20, 2021.

Publisher's Note Springer Nature remains neutral with regard to jurisdictional claims in published maps and institutional affiliations. 